\title{
Kyste dentigère infecté associé à une inclusion de canine maxillaire : à propos d'un cas
}

\section{An infected dentigerous cyst associated \\ with an impacted maxillary canine: a case report}

\section{Résumé}

\section{MOTS-CLEFS :}

- Kystes odontogéniques, kystes dentigères, dents incluses

\section{KEYWORDS:}

- Odontogenic cysts, dentigerous cysts, impacted teeth

$\operatorname{AOS} n^{\circ} 285-2017$
Les kystes dentigères, comme toute autre lésion kystique des maxillaires, sont des lésions bénignes qui peuvent présenter des signes cliniques différents selon la localisation et le stade d'évolution. Un kyste dentigère ou un kyste folliculaire est un kyste odontogène lié à une anomalie de développement de l'organe dentaire, Ce sont les plus fréquents des kystes odontogènes et ils sont liés généralement aux couronnes des dents qui n'ont pas encore fait leur éruption [1]. La cavité du kyste est bordée par des cellules épithéliales dérivées de l'épithélium adamantin réduit pendant la formation de l'organe de l'émail.

La pathogénie du kyste dentigère est liée ò la pression exercée par une dent en éruption sur le follicule pouvant obstruer la circulation veineuse induisant ainsi l'accumulation d'exsudat entre l'épithélium adamantin réduit et la couronne de la dent. [2]

Le cas présenté dans notre travail met en évidence l'importance ò accorder ò la démarche diagnostique des kystes des maxillaires, et plus particulièrement au kyste dentigère.
Abstract

The dentigerous cysts, like any other cystic lesion of the jaws, are benign lesions that mary present different clinical signs depending on the location and stage of development. À dentigerous cyst or follicular cyst is a cyst odontogenic linked to abnormal development of the dental organ, These are the most frequent odontogenic cysts and they are generally related to the crowns of the teeth that have not erupted [1]. The cyst cavity is lined by epithelial cells derived from the reduced enamel epithelium during the formation of the enamel organ.

The pathogenesis of dentigerous cyst is related to the pressure exerted by an erupting tooth on the follicle-clogging blood circulation and inducing accumulation of exudate between the reduced enamel epithelium and the crown of the tooth. [2]

The case presented in our work highlights the importance attached to the diagnostic process cysts of the jaws, particularly in dentigerous cyst.

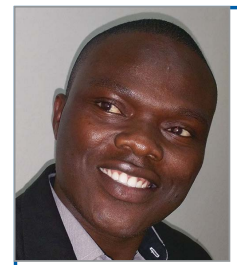

Bruno AFFOKPON, Résident en odontologie chirurgicale, Faculté de médecine dentaire de Rabat/MAROC, tél : +212 658033866, Email : jadearthur2013@gmail.com

Narjiss AKERZOUL, Résidente en odontologie chirurgicale.

Saliha CHBICHEB, Professeur d'enseignement supérieur en odontologie chirurgicale.

Wafaa EL WADY, Professeur d'enseignement supérieur en odontologie chirurgicale, chef de service d'odontologie chirurgicale. 


\section{INTRODUCTION}

Les pathologies radioclaires des maxillaires sont des lésions fréquentes de découverte fortuite le plus souvent à l'occasion d'un examen radiographique [2]. Elles font l'objet d'une classification codifiée qui entraine une méthodologie diagnostique minutieuse et une thérapeutique systématique.

Histologiquement, les kystes des maxillaires sont des lésions intraosseuses, odontogéniques ou non odontogéniques, possédant une structure histologique particulière caractérisée par la présence d'une enveloppe épithéliale, kératinisée ou non, et un contenu liquide, semi-liquide ou solide.

Selon la classification histopathologique de l'O.M.S basée sur l'étiologie et l'origine tissulaire, les kystes dentigères sont classés parmi les kystes épithéliaux odontogéniques (liés au développement) [3]. Le kyste dentigère est formé au sein de l'organe de l'émail d'une dent encore incluse, suite à l'accumulation de liquide kystique entre la couronne de la dent déjà formée et la paroi formée par les épithéliums adamantins interne et externe [4].

\section{OBSERVATION CLINIQUE ET DÉCISION THÉRAPEUTIQUE}

Un patient âgé de 47 ans, en bon état santé générale apparente, s'est présente en consultation pour une tuméfaction palatine localisée dans la région antérieure du maxillaire.

L'interrogatoire a révélé une tuméfaction évoluant depuis quelques semaines sans précision exacte de la part du patient.

À l'examen endobuccal, on découvre la présence d'une voussure palatine (Fig. 1), douloureuse à la pression, plus ou moins dépressible, une muqueuse de recouvrement normale, et l'absence de la canine maxillaire droite sur l'arcade.



$\triangle$ Fig. 1 : Vue pré-opératoire endobuccale montrant la voussure palatine en regard de la 13.
La radiographie panoramique, complétée par les radiographies occlusale, montre une image radio claire mono géodique englobant la couronne de la 13 qui est incluse en situation haute, faisant un angle de près de $120^{\circ}$ par rapport à l'axe vertical (Fig. 2). À la lumière $\mathrm{du}$ bilan clinique et radiographique, le diagnostic d'un kyste dentigère a été évoqué. D'autres lésions intra maxillaires ont été évoquées comme deuxième diagnostic. Il s'agit notamment de kératokyste odontogène, améloblastome unikystique, ou encore le kyste radiculo-dentaire.

Notre approche thérapeeutique a consisté en l'énucléation chirurgicale de la lésion avec l'extraction de la canine incluse.

L'anesthésie de la muqueuse palatine est réalisée par l'infiltration au niveau du foramen incisif, et au niveau de la 16 avec des rappels vestibulaires de 16 à 23 . Vu l'étendue de la lésion et la situation haute de la canine, le temps muqueux a consisté à faire une incision palatine de la 16 à la 23 au collet des dents avec un décollement prudent de la fibromuqueuse. Le temps osseux a consisté à une ostéotomie à l'aide d'une fraise à os boule montée sur pièce à main. L'énucléation du kyste a été réalisée, suivi de l'extraction de la 13 (Fig. 4a

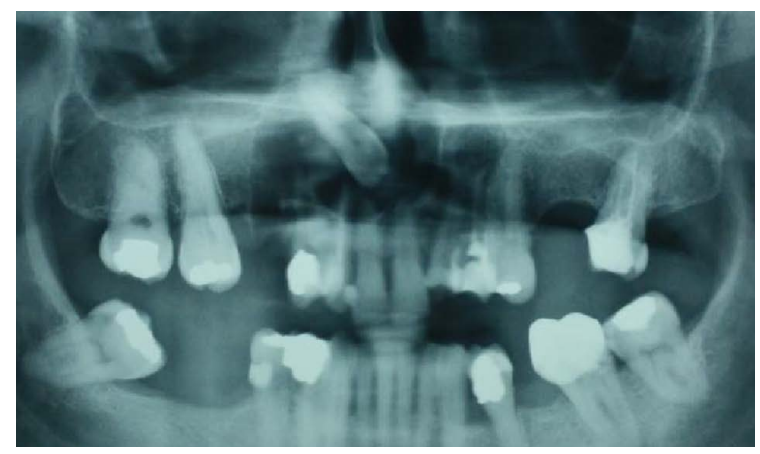

$\triangle$ Fig. 2 : La radiographie panoramique pré-opératoire, montrant l'image péri-coronaire entourant la couronne de la 13.



$\triangle$ Fig. 3 : Photo per-opératoire montrant l'énucléation kystique et l'extraction de la 13. 



$\triangle$ Fig. 4a et $4 \mathrm{~b}$ : À gauche, la cavité kystique après énucléation et extraction de la 13. À droite, la pièce opératoire et la canine extraite.

et $4 b)$. La cavité est soigneusement nettoyée et rincée avec du sérum physiologique. Ensuite le lambeau est remis en place et on a procédé à la réalisation des points de suture (Fig. 5).

La pièce est ensuite fixée dans du formol tamponné à $10 \%$ et envoyée pour examen anatomopathologique. La radiographie rétroalvéolaire réalisée en postopératoire 3 mois et radiographie panoramique réalisée après 6 mois ont montré un bon début de cicatrisation et de régénération osseuse (Fig. 6a et $\mathbf{6 b}$ ). L'examen anatomopathologique a confirmé le diagnostic de kyste dentigère.

\section{DISCUSSION}

Comme tout kyste des maxillaires, le kyste dentigère est une lésion ostéolytique. [5] D’origine odontogénique, anciennement appelé kyste folliculaire ou corono-dentaire, le kyste dentigère est observé à tout âge, avec une fréquence plus élevée entre la $2^{\mathrm{e}}$ et la $4^{\text {e }}$ décennie chez l'homme. Il intéresse surtout la $3^{\mathrm{e}}$ molaire mandibulaire, la canine maxillaire, la $2^{\mathrm{e}}$ prémolaire mandibulaire.

Le kyste dentigère peut provoquer une destruction osseuse (plus généralement la corticale externe des maxillaires), le déplacement des dents voisines et la résorption de leurs racines. Il empêche également l'éruption des dents permanentes associées au kyste. En denture temporaire, il est associé à un traumatisme, et est appelé kyste traumatique. Selon sa situation, il est soit central, soit péri-coronaire, soit latéral, soit peri-radiculaire ou circulaire. Le point d'insertion de la poche kystique est à la limite émail-cément [4]. Le kyste dentigère est souvent asymptomatique sauf en cas d'infection secondaire. De diagnostic tardif, il peut évoluer et prendre des proportions énormes. La radiographie panoramique montre une image radioclaire bien circonscrite, monogéodique avec liseré de condensation périphérique, englobant la couronne d'une dent incluse.. Un gros kyste dentigère peut



$\triangle$ Fig. 5 : Vue post-opératoire après suture.

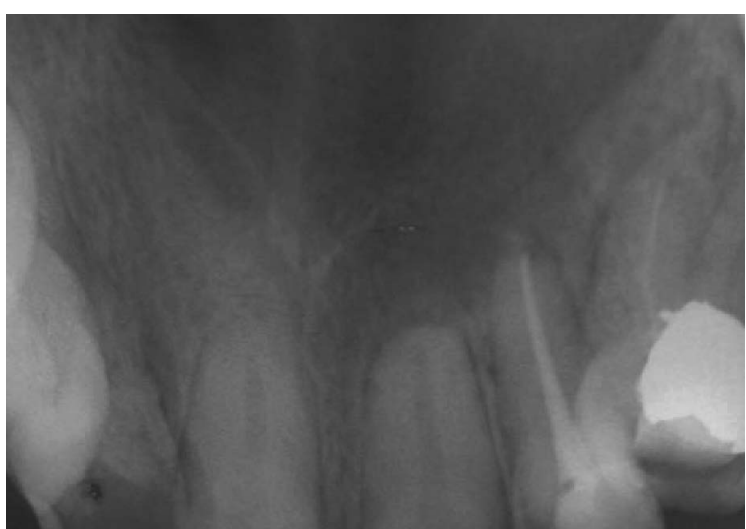

$\triangle$ Fig.6a: Vue post-opératoire endobuccale montrant la régénération osseuse après 3 mois.

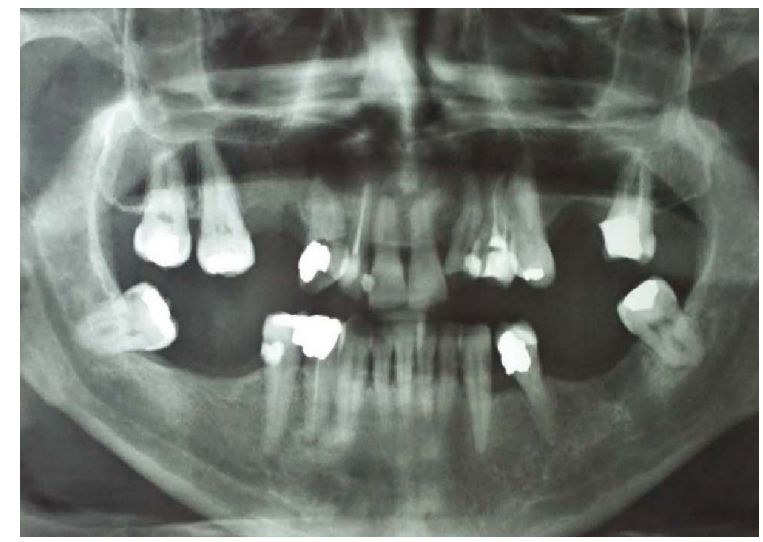

$\triangle$ Fig.6b : Vue panoramique post-opératoire montrant la régénération osseuse après 6 mois. 
donner l'impression d'un processus multiloculaire en raison de la persistance des travées osseuses au sein de la raréfaction.

Dans certains cas rapportés par des auteurs, on peut observer une résorption sur les dents adjacentes traduisant l'évolution lente et progressive du kyste [2], dans le cas présent aucune résorption radiculaire n'a été observée. Néanmoins on observe une rotation de la canine incluse dont la couronne se dirige vers la racine de l'incisive latérale et est oblique.

Le diagnostic différentiel se fait avec un améloblastome, un kératokyste, un kyste paradentaire. La confusion avec ces kystes se situe dans le fait que les images radiologiques des kystes dentigères évoquent un cloisonnement de la cavité kystique pouvant fausser le diagnostic. Un gros kyste dentigère peut donner l'impression d'un processus multiloculaire en raison de la persistance des travées osseuses au sein de la raréfaction. Cependant, les kystes dentigères sont généralement uniloculaires et probablement ne sont jamais vraiment des lésions multiloculaires. D'où l'importance d'un scanner pour mieux évaluer l'expansion du kyste et pour préciser son insertion au collet de la dent incluse [2]. Néanmoins certains éléments diagnostiques peuvent permettre de faire la différence entre les différents kystes.

L'aspect histologique de la paroi du kyste dentigère n'est pas toujours caractéristique. Le diagnostic repose donc principalement sur des éléments cliniques et radiologiques : image radiologique entourant la couronne et attachement du kyste à la jonction amélo-cémentaire de la dent associée ; l'absence de kératose à la surface de l'épithélium de la paroi kystique et de kératine dans la cavité kystique constituent également des éléments importants $[6,7]$. Le diagnostic différentiel doit faire évoquer :

I un sac péricoronnaire hyperplasique. Le diagnostic s'oriente vers un kyste dentigère s'il y a refoulement de la dent associée ou soufflure des corticales, ou si l'espace péricoronnaire dépasse une certaine épaisseur (8). Cette épaisseur varie selon les auteurs de 1 à $5 \mathrm{~mm}[6,7]$;

In kératokyste odontogène, mais il provoque moins de soufflure des corticales et de rhizalyses, et il ne s'attache jamais réellement à la jonction amélocémentaire ;

I un améloblastome unikystique ou un fibrome améloblastique qui, en raison de l'absence de structure interne, ne peuvent être différenciées radiologiquement ;

I un kyste radiculo-dentaire situé à l'apex d'une dent de lait et venant entourer la couronne de la dent définitive sous-jacente pour donner une image de kyste dentigère $[8]$; $\checkmark$ une tumeur adénomatoïde odontogène ou un kyste odontogène calcifié car ils peuvent simuler un kyste dentigère.

Très souvent solitaire, le kyste folliculaire peut être bilatéral ou multiple, retrouvé lors des syndromes tels que la dysostose cléidocranienne, le syndrome de Klippel-Feil [9].

Différentes possibilités thérapeutiques chirurgicales ont été appliquées pour traiter les kystes dentigères. Parmi elles, l'option conservatrice est très importante dans les cas de lésions de grands volumes et lorsque les dents permanentes concernées ont une grande potentialité éruptive [10]. Une autre possibilité thérapeutique consiste à mettre en place un dispositif dans la lumière kystique afin de maintenir une communication entre l'intérieur du kyste et la bouche. Cette procédure caractérise la décompression et diffère de la marsupialisation [11], car cette dernière consiste à former une poche communiquant avec l'extérieur en pratiquant une ouverture dans la cavité anatomique ou pathologique et en suturant le pourtour de cette ouverture aux lèvres de l'incision cutanée [12]. Les principaux avantages de la décompression ou de la marsupialisation de kystes dentigères sont entre autres le stimulus pour la formation de l'os après la diminution de la pression kystique ou encore la préservation des dents impliquées [13].

Le plus grand inconvénient du traitement conservateur est la préservation du tissu pathologique à l'intérieur des maxillaires. Le manque de coopération du patient dans le nettoyage de la cavité pathologique et la nécessité pour des visites de suivi périodiques représentent également des inconvénients supplémentaires. Malgré ce risque, la marsupialisation ou encore la décompression restent parmi les meilleures options thérapeutiques pour le patient quand le kyste est volumineux et avoisine des structures nobles : nerf dentaire inférieur, plancher nasal, sinus maxillaire car cela permet d'éviter de les léser et d'aboutir à des complications sur ces structures anatomiques.

La décision thérapeutique doit prendre en compte la possibilité carcinomateuse (exceptionnelle) de ce kyste et donc une énucléation complète de la poche kystique et de l'extraction de la dent incluse serait une solution non moins acceptable [2]

Dans notre cas, la décision d'extraction de la canine n'est pas facile, vu le rôle de pilier joué par la canine maxillaire dans la denture.

L'extraction de la canine maxillaire incluse est indiquée en cas de rotation de $180^{\circ}$ du germe, en cas d'ankylose aboutissant à l'échec du traitement endodontique, en cas de processus infectieux ou tumoral volumineux, ou en cas de refus du traitement orthodontique du patient. [13-14] 


\section{CONCLUSION}

Les kystes dentigères ont une évolution silencieuse et sont de découverte fortuite ou après extériorisation. On peut néanmoins la suspecter en cas d'absence d'éruption dentaire après le temps normal d'éruption sur l'arcade.

Le traitement chirurgical radical reste l'une des meilleures options pour éviter une récidive.

Lorsque le kyste dentigère est volumineux, l'énucléation doit être rigoureuse, pour éviter toute récidive, et faire l'objet d'un contrôle histologique attentif pour confirmer le diagnostic.

Lorsque le kyste dentigère est petit, il est de diagnostic et d'énucléation aisés.

Chez un sujet jeune, on peut envisager la marsupialisation lorsque le kyste dentigère est sur une canine ou une prémolaire pour favoriser l'évolution de la dent.

NB : cet article ne fait l'objet d'aucun conflit d'intérêt.

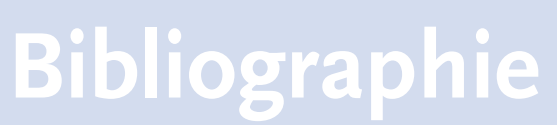

[1] Tilakraj Tn, et al. Non syndromic unilateral dentigerous cyst in a 4-year- old child : A rare case report. Contemp Clin Dent. 2011 Oct; 2(4):398-401.

[2] Thomas E. Les grands kystes des maxillaires : diagnostic, traitement et suivi. Thèse $n^{\circ}$ 3507, 2011.

[3] Sauveur G, et al. Kystes des maxillaires. Elsevier, 2006, 28-550-G-10.

[4] Perron JM. Accident d'évolution des dents de sagesse. Rouen : Elsevier SAS, 2004, vol 1, $\mathrm{n}^{\circ} 2, \mathrm{p}$ 147-158.
[5] K Mrad D. Kystes et tumeurs maxillo-mandibulaire, 2009. Actualités Odontostomatologique 2009 ; 252.

[6] White SC, et al. Oral radiology : principles and interpretation. Mosby Elsevier, St. Louis, 2009.

[7] Daley TD, et al. The small dentigerous cyst. À diagnostic dilemma. Oral Surg Oral Med Oral Pathol Oral Radiol Endod 1995 ; 79 : 77-81.

[8] Shear M, Speight PM. Cysts of oral and maxillofacialregions. Blacwell Munksgaard, 2007, 228p.

[9] Hyomoto M, et al. Clinical conditions for eruption of maxillary canines and mandibular premolars associated with dentigerous cysts. Am J Orthod Dentofac Orthop. 2003 ; 124 : 515-20.

[10] Horowitz RA, et al. Ultrastructural preservation of nuclei and chromatin : improvement with low-temperature methods. J Microsc. 1990 ; 157 : 205-24.

[11] Miyawaki S, et al. Eruption speed and rate of angulation change of a cystassociated mandibular second premolar after marsupialization of a dentigerous cyst. Am J Orthod Dentofac Orthop. 1999 ; 116 : 578-84.

[12] Baranes M, et al. Extraction des canines incluses. Actualités Odonto-stomatologiques $\mathrm{n}^{\circ} 244,2008$.

[13] Korbendau JM, et al. Réussir le traitement orthodontique et chirurgical des dents incluses. Quintessence International ; 2005.

[14] Bishara SE. Clinical management of impacted maxillary canines. Semin Ortho 1998 ; $4(2)$ : 87-98. 